J. E. Cecil • B. Fischer - A. S. F. Doney •

M. Hetherington $\cdot$ P. Watt $\cdot$ W. Wrieden $\cdot$

C. Bolton-Smith · C. N. A. Palmer

\title{
The Pro12Ala and C-681G variants of the PPARG locus are associated with opposing growth phenotypes in young schoolchildren
}

Received: 30 November 2004 / Accepted: 4 April 2005 / Published online: 9 July 2005

(C) Springer-Verlag 2005

\begin{abstract}
Aims/hypothesis: Peroxisome proliferator-activated receptor $\gamma$ is an important regulator of adiposity in mouse and man, and common variation in the PPARG gene has been associated with birthweight, adult obesity, insulin sensitivity and type 2 diabetes. We hypothesised that these variants may be associated with childhood obesity. Methods: Height and weight were recorded for 2454 prepubertal children aged between 4 and 10 years, who were then genotyped for three common variants of the PPARG locus: C-681G, Pro12Ala and C1431T. Results: No single variant of $P P A R G$ was significantly associated with height, weight or BMI. However, when modelling the variants together we detected an opposing interaction between the $-681 \mathrm{G}$ and the Ala12 variants in height and weight, but not BMI ( $p=0.018,0.013$ and 0.119 respec-
\end{abstract}

\footnotetext{
J. E. Cecil

The Bute Medical School, University of St Andrews,

St Andrews, Scotland, UK

B. Fischer · A. S. F. Doney · C. N. A. Palmer $(\bowtie)$

Biomedical Research Centre, Ninewells Hospital

and Medical School, University of Dundee,

Dundee, Scotland, UK

e-mail: nuclear-receptor@dundee.ac.uk

Tel.: +44-1382-632744

Fax: +44-1382-669993

M. Hetherington

Department of Psychology, University of Liverpool,

Liverpool, UK

P. Watt

Department of Sport and Exercise Science,

Chelsea School, University of Brighton,

Brighton, UK

\author{
W. Wrieden \\ Dundee, Scotland, UK \\ C. Bolton-Smith \\ MRC Human Nutrition Unit, \\ Cambridge, UK
}

Centre for Public Health Nutrition Research,

Ninewells Hospital and Medical School,
}

tively). The data were consistent with the Ala12 carriers being deficient in energy storage/utilisation, leading to reduced growth. In contrast, the $-681 \mathrm{G}$ variant, which has been associated with increased adult height, was associated with accelerated growth. The two variants were in strong linkage disequilibrium. However, rare individuals bearing the isolated variants demonstrated the greatest variation from the mean, the most contrasting genotypes being associated with a variation of $7 \mathrm{~kg}$ in weight and $6 \mathrm{~cm}$ in height, standardised to 7.4-year-olds ( $p=0.006$ and $p=0.02$ espectively). Conclusions/interpretation: This study demonstrates that quantitative trait analysis of energy balance/ growth and the PPARG locus is complex and requires the use of multiple genetic markers.

Keywords Body mass - Children · Growth · Height · Human genetics · Obesity

Abbreviations PPAR: peroxisome proliferator-activated receptor

\section{Introduction}

The epidemic of obesity that is occurring throughout the world is reflected in an increasing prevalence of childhood obesity [1-3]. Dietary factors and sedentary lifestyles combine to promote positive energy balance, resulting in excess energy intake relative to requirements and the deposition of fat in adipose stores. It has been proposed that there is a strong genetic component in the tendency to increased adiposity in the face of caloric excess. This is known as the 'thrifty genotype' [4]. A strong candidate gene for a component of this thrifty genotype is PPARG [5]. PPARG encodes peroxisome proliferator-activated receptor $\gamma(\operatorname{PPAR} \gamma)$, a nuclear receptor that senses fatty acids and controls the programme of gene expression required for the formation of new adipocytes. Studies in mice deleted for the PPARG gene have shown that $P P A R G$ is essential for the formation of adipose tissue [6], and humans who are heterozygous for dominant-negative variants of $P P A R G$ display partial 
peripheral lipodystrophy [7-9]. Common variants of this gene have been described and have been associated with various traits related to obesity. The most studied of these is the substitution of alanine for proline at codon 12 of PPAR $\gamma 2$ [10]. The proline form is the most common and is associated with susceptibility to the development of type 2 diabetes $[11,12]$. This variant is associated with higher levels of insulin and a corresponding increase in weight, although reports of the associations with altered BMI have been conflicting. This may be due to genegene and gene-environment interactions, in addition to the action of additional variants in the PPARG gene itself $[13,14]$. The penetrance of the Ala12 variant appears to interact with diet, in the form of both fat [15-17] and carbohydrate intake [18]. The proline variant also appears to interact with the response to exercise, with increased body weight in response to training, whereas individuals with the alanine variant respond poorly to training, with a marked loss of weight [14]. This is supported by a recent study that demonstrated an interaction between Ala12, diet and exercise in the control of insulin secretion [19].

The effect of the variation at codon 12 may be modulated by its chromosomal context. Other variants have been found to be in linkage disequilibrium with this variant, and together these have been associated with diabetesrelated traits, which modulate the associations observed with the codon 12 substitution. In particular, the C1431T variant, which is a synonymous codon change in exon 6 , has been associated with increased body mass [20], altered BMI/leptin ratios [21] and susceptibility to cardiovascular disease $[22,23]$. We have previously presented data indicating that the association of the codon 12 substitution with body weight and susceptibility to type 2 diabetes is modulated by its context with respect to this C1431T variant $[13,14]$. Recently a third polymorphism in strong linkage disequilibrium with the codon 12 variant has been described $(\mathrm{C}-681 \mathrm{G})$ [24]. This variant resides in the PPAR $\gamma 3$ promoter region and is used in a range of nonadipose cells, including bone marrow-derived leucocytes. The $\mathrm{C}-681 \mathrm{G}$ variant affects the binding of the transcription factor STAT5 (signal transducer and activator of transcription 5) and has been proposed to modulate the regulation of PPAR $\gamma$ expression by growth hormone, which may in turn regulate bone growth. The $-681 \mathrm{G}$ variant has been associated with increased height in an adult population, providing support for this hypothesis. However, detailed mechanistic studies regarding PPAR $\gamma$ and growth have not been performed. The role of PPAR $\gamma$ as a regulator of growth has been established recently in pparg $+1-$ mice, in which lean mass growth was reduced, and IGF-1 production in white adipose tissue was attenuated [25]. However, this study did not examine the possibility of altered growth hormone signalling in muscle or bone.

Most work on genetic associations with body weight and variation at the PPARG locus has focused on adults. However, recent studies have presented evidence that $P P A R G$ is involved in birth size [26], and therefore PPARG will have a role in energy balance throughout life. In this study we explore the role of the PPARG locus in the regulation of growth in a large cohort of young primary school children $(n=2454)$, and we find that the PPARG locus has a role in modulating prepubescent growth. This modulation is dependent on the haplotype structure of the PPARG locus and is consistent with the emerging hypotheses regarding the role of specific variants in adult energy balance.

\section{Subjects and methods}

Subject recruitment Subjects were healthy prepubertal schoolchildren, recruited through 47 primary schools in the Tayside region of East Scotland $(n=2454)$. The sample was largely Caucasian (98\%). All children aged between 4 and 10 years were invited to participate in the study, through letters and information sheets distributed in the schools to parents and guardians. Parents provided written informed consent for their child's participation. Researchers visited each school and genomic DNA isolated from saliva was taken from the consenting sample. Standing height, without shoes, was measured (to the nearest $0.1 \mathrm{~cm}$ ) using a stadiometer (Seca Leicester height measure). Body weight was measured (to the nearest $0.1 \mathrm{~kg}$ ) using a mechanical scale (Seca mechanical floor scale) with subjects wearing light clothing. BMI was calculated as weight/ height ${ }^{2}\left(\mathrm{~kg} / \mathrm{m}^{2}\right)$. Date of birth was also obtained. Ethical approval was granted from the Tayside Committee on Medical Research Ethics and the Fife Local Research Ethics Committee. In addition, the Education Department of each school authority approved the study.

DNA preparation and genotyping DNA was prepared from mouthwash pellets as previously described [27]. The failure rate for DNA preparation was less than $0.1 \%$, and failure was associated with small cellular pellets from younger children (4 to 5-year-olds), who had difficulty spitting. Genotyping was performed using Taqman-based allelic discrimination assays, analysed on an ABI 7700 (Applied Biosystems, Foster City, CA, USA). The Pro12Ala and $\mathrm{C} 1431 \mathrm{~T}$ variants were genotyped as described previously [14]. The $\mathrm{C}-681 \mathrm{G}$ polymorphism was genotyped using the following probes and primers: primer 1, CTGA TGATAAGGCTTTTGGCATT; primer 2, CTCTTAT GAA AGGCTCAAGGATCC; probe 1 (Fam-labelled), ATGCT GTTTTGTCTTCATGGAAAATACAGCTATTCT; probe 2, (Tet-labelled), ATGCTGTTTTGTCTTGATGGAAAATA CAGCTATTCT.

All three genotyping assays provided genotypes for more than $98 \%$ of the total population, and intra-individual variation of replicate genotypes was greater than $99 \%$. Three sample replicates of known genotype were included on each plate to monitor genotyping accuracy and to confirm cluster analysis.

Statistical analysis Haplotype frequencies were determined statistically using PHASE version 1.0.1 under Mac OS X [28]. D' was calculated using the 2-LD program [29]. 

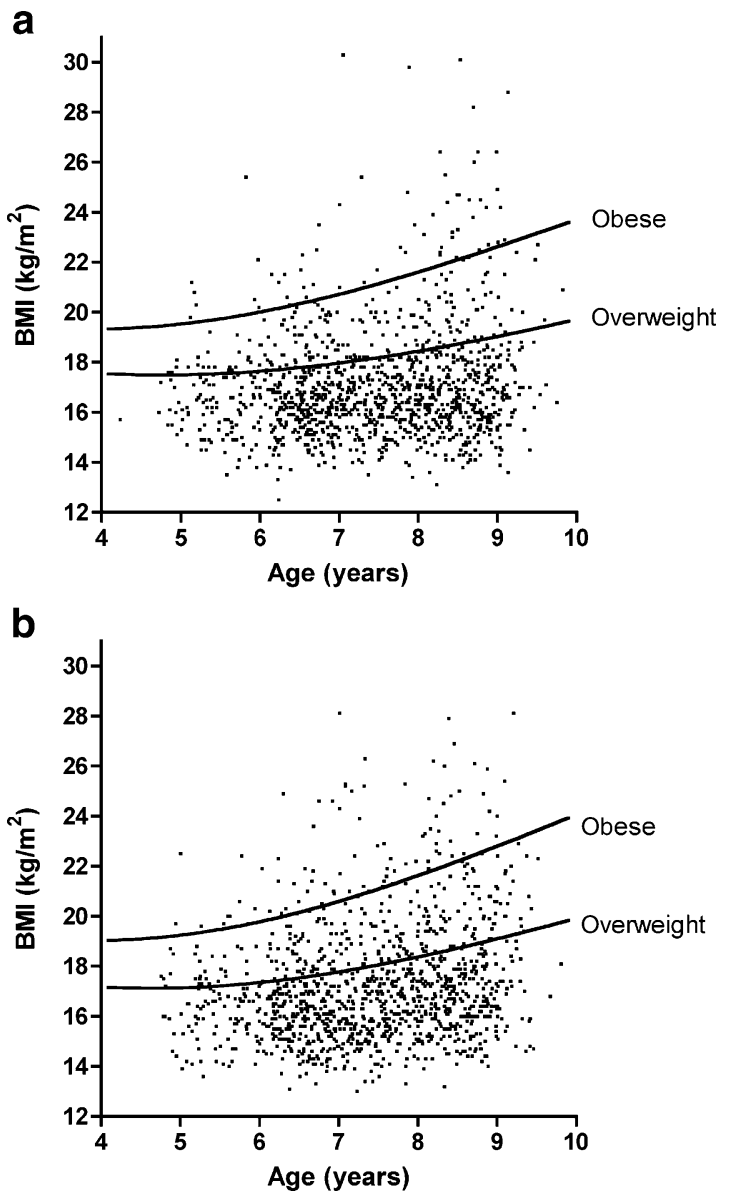

Fig. 1 Prevalence of overweight and obese children in the cohort. Distribution plots of BMI vs age for males (a) and females (b). The standard curves for obesity and overweight specific to each sex are shown. These curves were derived from a summary of international cohorts

Quantitative traits (height, weight and BMI) were analysed using univariate general linear modelling with SPSS version 11.5 for Windows (SPSS, Chicago, IL, USA). Age and sex were included in all models. The role of the individual variants was assessed by step-wise removal. This resulted in a final model containing only the $-681 \mathrm{G}$

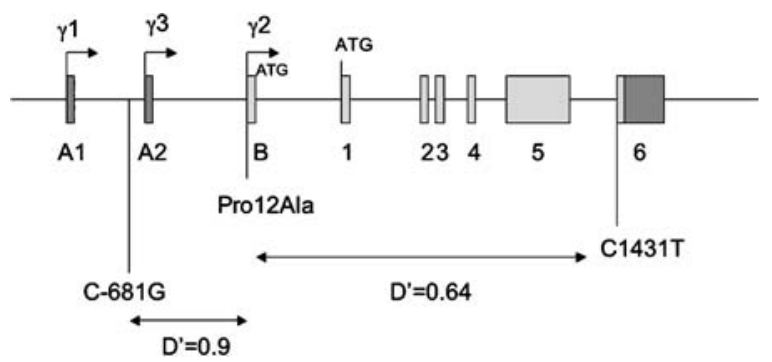

Fig. 2 A schematic illustration of the genomic context of the PPARG variants. The transcribed regions are shown as boxes, coding sequences in light grey and non-coding sequences in dark grey. $\gamma 1, \gamma 2$ and $\gamma 3$ represent alternative $5^{\prime}$ exons derived from different promoters
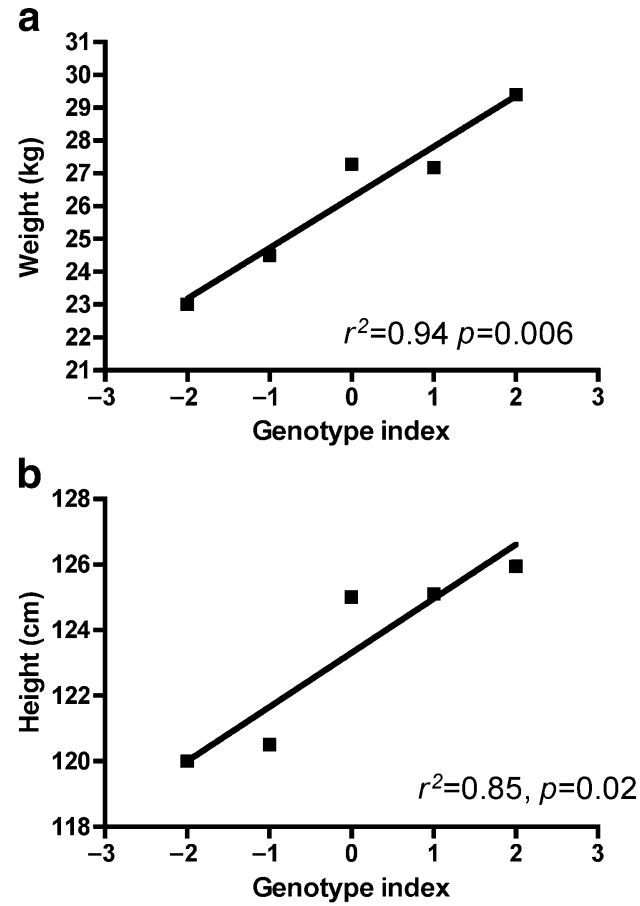

Fig. 3 Opposing associations of Pro12Ala and C-681G with height and weight. Individuals were scored with -1 per copy of the Ala12 variant and +1 per copy of the $-681 \mathrm{G}$ variant to derive a genotype index. This genotype index was used as an ordinal to plot against estimated means of weight (a) and height (b). The resulting plots were analysed by linear regression and the resulting $r^{2}$ and $p$ values are shown. Numbers of individuals in each group: genotype index $-2, n=1$; index $-1, n=12$; index $0, n=1888$; index $+1, n=481$; index $+2, n=31$

and Ala12 variants. Both recessive and co-dominant genetic models were tested. Standard deviation scores for height, weight and BMI were generated relative to the 1990 British growth reference charts [30,31]. All $p$ values are two-way. A $p$ value of $<0.05$ was considered significant. Correction for multiple testing was not applied, as only primary hypotheses were tested and the PPARG locus has been associated with energy utilisation and obesity in many studies. The graphs shown in Figs. 1, 2, 3, 4 were prepared using Graphpad Prism for Macintosh, version 4 (GraphPad, San Diego, CA, USA).

\section{Results}

The height, weight and BMI was determined in 2454 children (Table 1) and compared with an international standard for overweight and obesity [32]. In this cohort there were $5.0 \%$ obese boys and $7.2 \%$ obese girls. In addition, $21.1 \%$ of the boys and $28.1 \%$ of the girls were overweight or obese (Fig. 1). This represents a markedly higher prevalence of obesity and overweight in both sexes compared with a 1994 survey of Scottish children of similar age range [3].

The allele frequencies of all three $P P A R G$ variants were similar to those reported for other Caucasian populations 

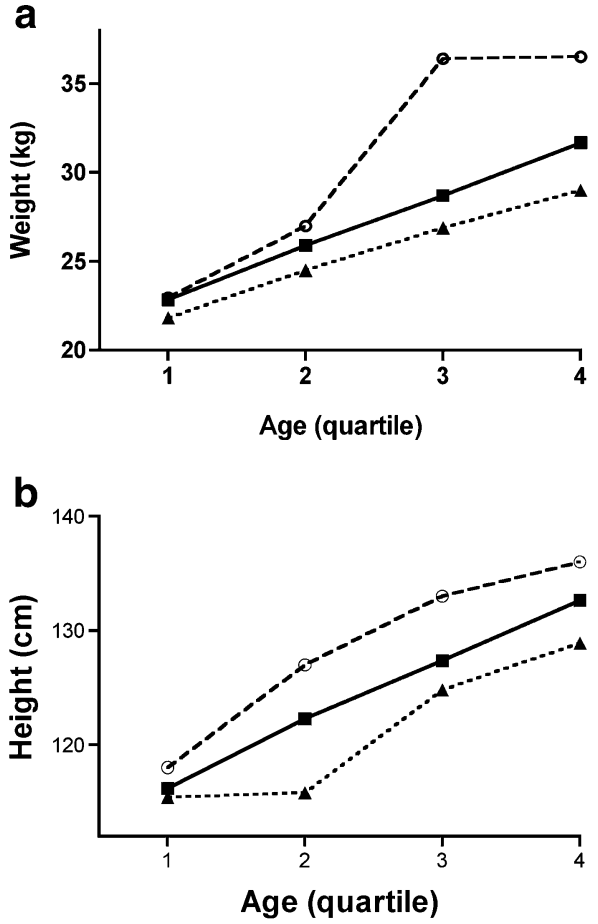

Fig. 4 Opposing associations of Pro12Ala and C-681G with growth. The population was categorised for quartiles of age and the resulting quartile value was used as an ordinal value. Estimated means of weight (a) and height (b) were plotted against the resulting ordinal values. Individuals with only the Ala12 variant (one or two copies, $n=13$ ) are represented by triangles, individuals carrying only the common variants Pro 12 and $-681 \mathrm{C}(n=1410)$ are represented by squares, and individuals carrying only the $-681 \mathrm{G}$ variant (one or two copies, $n=442$ ) are represented by open circles. The quartiles of age were: $1,4.23-6.59$ years; $2,6.59-7.50$ years; $3,7.50-8.36$; 4 , $8.36-9.83$ years

(Table 2). The strong linkage disequilibrium between the $-681 \mathrm{G}$ and the Ala12 variants (Fig. 2, Table 3) results in very few Ala12 carriers that do not carry the $-681 \mathrm{G}$; however, the greater frequency of the $-681 \mathrm{G}$ variant means that a substantial number of individuals carry the $-681 \mathrm{G}$ without Ala12 (Table 3). This allows us to examine the role of this variant in isolation.

We initially examined the role of the individual variants in determining height, weight and BMI in the children. We used univariate general linear modelling and found that sex and age were major determinants of all three parameters, as would be expected (Table 4). Therefore sex and age were retained in the model. All three variants were

Table 1 Summary of population characteristics

\begin{tabular}{llll}
\hline & Mean & Minimum & Maximum \\
\hline Age (years) & 7.4 & 4.2 & 9.8 \\
Height $(\mathrm{cm})$ & 125.2 & 101.4 & 150.3 \\
Weight $(\mathrm{kg})$ & 27 & 14 & 65 \\
BMI $\left(\mathrm{kg} / \mathrm{m}^{2}\right)$ & 17.2 & 10.8 & 30.3 \\
$n=2454$ & 1240 male & 1214 female & \\
\hline
\end{tabular}

Table 2 Genotypes of study population

\begin{tabular}{lrrl}
\hline Polymorphism & $n$ & Genotype frequency (\%) & Allele frequency \\
\hline Pro12Ala & & & \\
Pro/Pro & 1866 & 76.7 & 0.125 \\
Pro/Ala & 528 & 21.7 & \\
Ala/Ala & 39 & 1.6 & \\
C1431T & & & 0.132 \\
C/C & 1835 & 75.2 & \\
C/T & 568 & 23.3 & \\
T/T & 38 & 1.6 & 0.300 \\
C-681G & & & \\
C/C & 1425 & 58.8 & \\
C/G & 862 & 35.6 & \\
G/G & 137 & 5.7 & \\
\hline
\end{tabular}

included in the model and interactions between these variants were tested. Using this model, none of the variants associated individually with height, weight or BMI. However, a gene dose-dependent trend was observed with Pro12Ala and C-681G on height and weight, Ala12 being associated with reduced height and weight and $-681 \mathrm{G}$ being associated with increased height and weight (Table 4). The tests for interactions revealed a consistent and statistically significant interaction with Pro12Ala and C-681G for all heights and weights, with the strongest interaction being for weight.

As the C1431T polymorphism had very little effect in this model, we repeated the test with this variant removed. This resulted in the Pro12Ala polymorphism becoming significantly associated with reduced weight and height $\left(p^{2}\right.$, Table 4); C-681G alone remained insignificant but the interaction between $\mathrm{C}-681 \mathrm{G}$ and Pro12Ala was significant. Examination of the individual variants in isolation did not provide any significant associations with height, weight or BMI (data not shown). The relationships between height, weight, BMI and age are highly non-linear up to the age of 10 years; therefore, there was a possibility that the observed genotypic effects were subject to error in the adjustment for age and sex. We therefore analysed the same model for C-681G and Pro12Ala using standard deviation scores of height, weight and BMI, based on the UK reference growth charts (Table 5), which resulted in outcomes similar to those obtained using age as a covariate in the analysis of the raw measures.

As the Pro12Ala and C-681G variants appeared to oppose each other and an interaction was indicated by the general linear modelling, we visualised the relationship of the two variants based on an allele scoring scheme (genotype index). As the Ala12 variant was associated with reduced weight, a chromosomal copy of the Ala12 was scored as -1 ; and as the $-681 \mathrm{G}$ was associated with increased height and weight, a single copy was scored as +1 . Therefore homozygote Ala12 individuals with no $-681 \mathrm{G}$ scored as -2 , homozygote $-681 \mathrm{G}$ individuals with no Ala12 scored as +2 , and dual homozygote Ala12/-681G individuals scored as 0 . The weights and heights of all 
Table 3 Haplotype frequencies and linkage disequilibrium

\begin{tabular}{lll}
\hline Haplotype & Haplotype no. & Frequency \\
\hline -681C,Pro12,C1431 (wild-type) & 1 & 0.734 \\
$-681 \mathrm{G}$, Pro12,C1431 ( $\gamma 3$ promoter alone) & 2 & 0.099 \\
$-681 \mathrm{G}$, Ala12,T1431 (all three variants) & 3 & 0.087 \\
$-681 \mathrm{G}$, Ala12,C1431 & 4 & 0.035 \\
$-681 \mathrm{C}$, Pro12,T1431 (T1431 alone) & 5 & 0.030 \\
$-681 \mathrm{G}$, Pro12,T1431 & 6 & 0.013 \\
$-681 \mathrm{C}$, Ala12,T1431 & 7 & 0.0014 \\
$-681 \mathrm{C}$, Ala12,C1431 (Ala12 alone) & 8 & 0.0014 \\
\hline Linkage disequilibrium & $\mathrm{D}^{\prime}$ & $r^{2}$ \\
\hline Ala12 vs -681G & 0.97 & 0.52 \\
Ala12 vs T1431 & 0.64 & 0.53 \\
$-681 G$ vs T1431 & 0.65 & 0.43 \\
\hline
\end{tabular}

children were adjusted to a mean age of 7.4 years and for sex, and were plotted against the genotype index as an ordinal value (Fig. 3). This characterises the dose-response of the genetic interaction between these two variants, with an almost $7 \mathrm{~kg}$ difference in weight and a $6 \mathrm{~cm}$ difference in height between the most contrasting genotypes $(p=0.006$ and $p=0.02$ respectively).

As the data reflect a growing population, with children at various ages, we explored the hypothesis that the variants affect the growth of children. This was characterised by plotting the height and weights by quartile of age (Fig. 4). This analysis demonstrates that the genotype effect was consistent across the age groups, the $-681 \mathrm{G}$ being associated with accelerated growth and the Ala12 being associated with reduced growth compared with individuals bearing the common Pro12/-681C variants, who were intermediate in growth. Both height and weight were associated with both polymorphisms in a concerted fashion, and this was consistent with an effect on growth rather than adiposity, indicating that the polymorphisms are associated with body size.

As our study included a substantial number of obese and overweight children, we also explored the relationship between these alleles and susceptibility to obesity. The frequencies of the $\mathrm{T} 1431$ and $-681 \mathrm{G}$ variants were not altered in any of the quartiles of BMI (data not shown). However, the Ala12 carrier frequency showed a significant trend in depletion towards the higher quartiles of BMI (Fig. 5, $p=0.026$ ), supporting the hypothesis that this variant protects from childhood obesity.

\section{Discussion}

The PPARG locus has been studied extensively for traits associated with diabetes and obesity in adults. The emerging consensus is that the Ala12 variant confers a subtle protection from obesity, insulin resistance and type 2 dia-

Table 4 Association of PPARG variants with height, weight and BMI

\begin{tabular}{lllllllllllllll}
\hline Codon 12 & Mean & SEM & $p^{1}$ & $p^{2}$ & C1431T & Mean & SEM & $p^{1}$ & $p^{2}$ & C-681G & Mean & SEM & $p^{1}$ & $p^{2}$ \\
\hline BMI & & & & & & & & & & & & & & \\
Pro/Pro & 17.7 & 0.32 & & & C/C & 16.9 & 0.41 & & & C/C & 17.2 & 0.53 & & \\
Pro/Ala & 16.9 & 0.26 & & & C/T & 17.4 & 0.35 & & & C/G & 16.6 & 0.46 & & \\
Ala/Ala & 16.6 & 0.69 & 0.337 & 0.113 & T/T & 17.2 & 0.52 & 0.800 & N/A & G/G & 17.7 & 0.27 & 0.103 & 0.402 \\
Weight & & & & & & & & & & & & & & \\
Pro/Pro & 28.5 & 0.7 & & & C/C & 26.3 & 0.9 & & & C/C & 26.5 & 1.2 & & \\
Pro/Ala & 26.4 & 0.6 & & & C/T & 27.7 & 0.8 & & & C/G & 26.0 & 1.0 & & \\
Ala/Ala & 25.8 & 1.6 & 0.267 & 0.013 & T/T & 27.0 & 1.2 & 0.804 & N/A & G/G & 28.2 & 0.6 & 0.150 & 0.117 \\
Height & & & & & & & & & & & & & & \\
Pro/Pro & 125.5 & 0.8 & & & C/C & 124.0 & 0.9 & & & C/C & 122.9 & 1.3 & & \\
Pro/Ala & 124.1 & 0.6 & & & C/T & 125.5 & 0.8 & & & C/G & 124.8 & 1.0 & & \\
Ala/Ala & 124.0 & 1.7 & 0.566 & 0.019 & T/T & 124.1 & 1.3 & 0.771 & N/A & G/G & 125.7 & 0.7 & 0.334 & 0.044 \\
\hline
\end{tabular}

Shown are the estimated means adjusted for age and sex

$p^{1}$, model including C1431T; $p^{2}$, model excluding C1431T. No significant values were obtained with individual variants in the model Recessive and co-dominant models were tested; the best model was a co-dominant action of all variants and the $p$ values shown were derived using this model

$p=0.119$ for interaction between Pro12Ala and C-681G (BMI)

$p=0.013$ for interaction between Pro12Ala and C-681G (Weight)

$p=0.018$ for interaction between Pro12Ala and C-681G (Height) 
Table 5 Association of PPARG variants with deviation from UK standard growth charts

\begin{tabular}{|c|c|c|c|c|c|c|c|}
\hline Codon 12 & Mean & SEM & $p$ & $\mathrm{C}-681 \mathrm{G}$ & Mean & SEM & $p$ \\
\hline \multicolumn{8}{|l|}{ BMI-SDS } \\
\hline Pro/Pro & 0.79 & 0.06 & & $\mathrm{C} / \mathrm{C}$ & 0.45 & 0.35 & \\
\hline Pro/Ala & 0.58 & 0.11 & & $\mathrm{C} / \mathrm{G}$ & 0.52 & 0.24 & \\
\hline Ala/Ala & 0.35 & 0.41 & 0.180 & $\mathrm{G} / \mathrm{G}$ & 0.76 & 0.09 & 0.476 \\
\hline \multicolumn{8}{|c|}{ Weight-SDS } \\
\hline Pro/Pro & 0.72 & 0.7 & & $\mathrm{C} / \mathrm{C}$ & -0.05 & 0.36 & \\
\hline Pro/Ala & 0.41 & 0.6 & & $\mathrm{C} / \mathrm{G}$ & 0.48 & 0.24 & \\
\hline Ala/Ala & 0.01 & 1.6 & 0.021 & $\mathrm{G} / \mathrm{G}$ & 0.71 & 0.09 & 0.093 \\
\hline Codon 12 & Mean & SEM & $p^{1}$ & $\mathrm{C}-681 \mathrm{G}$ & Mean & SEM & $p^{1}$ \\
\hline \multicolumn{8}{|c|}{ Height-SDS } \\
\hline Pro/Pro & 0.30 & 0.06 & & $\mathrm{C} / \mathrm{C}$ & -0.58 & 0.35 & \\
\hline Pro/Ala & -0.02 & 0.11 & & $\mathrm{C} / \mathrm{G}$ & 0.19 & 0.24 & \\
\hline Ala/Ala & -0.37 & 0.41 & 0.021 & $\mathrm{G} / \mathrm{G}$ & 0.31 & 0.09 & 0.048 \\
\hline
\end{tabular}

Shown are the estimated means of the standard deviation scores

(SDS) derived from the British 1990 reference growth charts [30, 31]

$p=0.242$ for interaction between Pro12Ala and C-681G (BMI-SDS) $p=0.046$ for interaction between Pro12Ala and C-681G (WeightSDS)

$p=0.020$ for interaction between Pro12Ala and C-681G (HeightSDS)

betes. The Ala12 variant also appears to be pleiotropic and is associated with protection from other obesity-associated diseases, such as hypertension and myocardial infarction [33-35]. It is therefore important to note that we can observe significant differences in susceptibility to obesity associated with this variant even at this early age. The magnitude of the effects on BMI and obesity are much smaller than we have observed in three adult populations [14], and this may reflect the fact that PPAR $\gamma$ is mainly affecting growth in this population. The main site of PPAR $\gamma$ action in growth is unclear; however, it is possible that the

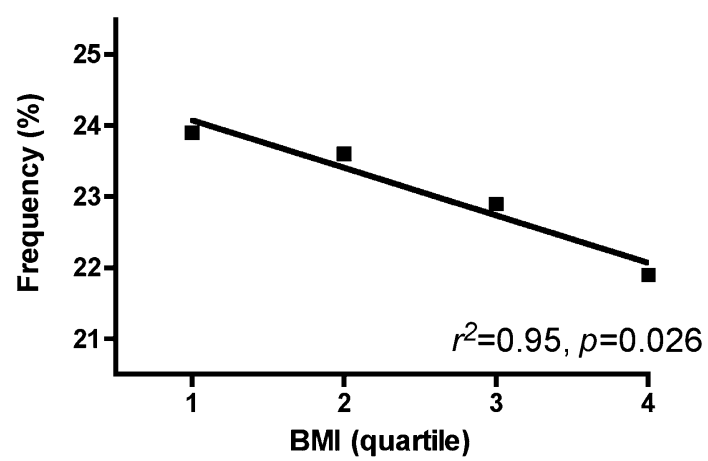

Fig. 5 The Ala12 variant is depleted in obesity. The population was characterised for quartiles of BMI and the resulting quartile values were used as an ordinal value. The Ala12 carrier frequency was plotted against this ordinal value of BMI and analysed by linear regression. The resulting $r^{2}$ and $p$ values are shown. The quartiles of BMI were: $1,10.8-15.6 \mathrm{~kg} / \mathrm{m}^{2} ; 2,15.7-16.7 \mathrm{~kg} / \mathrm{m}^{2} ; 3,16.8-18.1$ $\mathrm{kg} / \mathrm{m}^{2} ; 4,18.2-30.3 \mathrm{~kg} / \mathrm{m}^{2}$
Ala12 variant affects insulin secretion and may therefore indirectly affect muscle and bone growth. The $-681 \mathrm{G}$ polymorphism, on the other hand, has been postulated to interfere directly with growth factor signalling in the bone [24]; however, exact mechanistic details of this have not been demonstrated in vivo. This study represents an important extension of the initial report of this variant, where it was associated with adult height and not BMI. The lack of effect of the C1431T variant does not appear to reflect the inclusion of the $-681 \mathrm{G}$ variant in the model, as the C1431T did not associate with any parameters on its own or in any combination of the other variants. This is in contrast to our previous reports on several populations of adults $[13,14]$. It is possible that the impact of this variant on BMI may become apparent later in life. As yet no functional mechanism has been given to this variant, although we consistently find diabetes-related traits associating in adults with this variant in opposition to the Ala12 variant $[13,36]$.

This investigation forms part of a large population-based study in which we have looked at the interactions of haplotypes of three variants in one locus, and it is clear that most of the signal obtained is from rare haplotypes in which the variants are dissociated. This is problematic for smaller studies, in which these rare haplotypes will be extremely sparse and may be completely absent. In addition, as the main signal is obtained from rare haplotypes, it is possible that population stratification may be responsible for the observed result. Examination of the non-Caucasian subgroup $(n=50)$ in this study revealed that the allele frequency, linkage disequilibrium, height for age and weight for age were all similar between both cohorts, and that the rare haplotypes were only present in the Caucasian sample; therefore, gross ethnic differences were not responsible for the observed genetic effect. In fact, similar trends by genotype in both cohorts were apparent, the interactions in the Caucasian cohort remaining significant. The allele frequencies did not differ in age group or by sex, and therefore these parameters did not confound this study.

Finally, although we have found an association with apparent growth, this is based on single measures of individuals within a population. It will be important to follow a genetically enriched subpopulation and to examine the energy balance/growth characteristics of these individuals throughout their entire growing period.

Acknowledgements We thank Ms I. Murrie and Ms D. Wallis for their help in the sample collection. This study was funded by the Biotechnology and Biological Sciences Research Council project grant D13460. C.N.A. Palmer is supported by the Scottish Executive Genetic Health Initiative.

\section{References}

1. Lobstein T, Frelut ML (2003) Prevalence of overweight among children in Europe. Obes Rev 4:195-200

2. Lobstein TJ, James WP, Cole TJ (2003) Increasing levels of excess weight among children in England. Int J Obes Relat Metab Disord 27:1136-1138 
3. Chinn S, Rona RJ (2001) Prevalence and trends in overweight and obesity in three cross sectional studies of British Children, 1974-94. BMJ 322:24-26

4. Neel JV (1962) Diabetes mellitus: a 'thrifty' genotype rendered detrimental by 'progress'? Am J Hum Genet 14:353-362

5. Auwerx J (1999) PPARgamma, the ultimate thrifty gene. Diabetologia 42:1033-1049

6. Rosen ED, Hsu CH, Wang X et al (2002) C/EBPalpha induces adipogenesis through PPARgamma: a unified pathway. Genes Dev 16:22-26

7. Agarwal AK, Garg A (2002) A novel heterozygous mutation in peroxisome proliferator-activated receptor-gamma gene in a patient with familial partial lipodystrophy. J Clin Endocrinol Metab 87:408-411

8. Hegele RA, Cao H, Frankowski C, Mathews ST, Leff T (2002) PPARG F388L, a transactivation-deficient mutant, in familial partial lipodystrophy. Diabetes 51:3586-3590

9. Savage DB, Tan GD, Acerini CL et al (2003) Human metabolic syndrome resulting from dominant-negative mutations in the nuclear receptor peroxisome proliferator-activated receptorgamma. Diabetes 52:910-917

10. Yen CJ, Beamer BA, Negri C et al (1997) Molecular scanning of the human peroxisome proliferator activated receptor gamma (hPPAR gamma) gene in diabetic Caucasians: identification of a Pro12Ala PPAR gamma 2 missense mutation. Biochem Biophys Res Commun 241:270-274

11. Deeb SS, Fajas L, Nemoto M et al (1998) A Pro12Ala substitution in PPARgamma2 associated with decreased receptor activity, lower body mass index and improved insulin sensitivity. Nat Genet 20:284-287

12. Altshuler D, Hirschhorn JN, Klannemark M et al (2000) The common PPARgamma Pro12Ala polymorphism is associated with decreased risk of type 2 diabetes. Nat Genet 26:76-80

13. Doney AS, Fischer B, Cecil JE et al (2004) Association of the Pro12Ala and C1431T variants of PPARG and their haplotypes with susceptibility to type 2 diabetes. Diabetologia 47:555-558

14. Doney A, Fischer B, Frew D et al (2002) Haplotype analysis of the PPARgamma Pro12Ala and C1431T variants reveals opposing associations with body weight. BMC Genet 3:21

15. Robitaille J, Despres JP, Perusse L, Vohl MC (2003) The PPAR-gamma P12A polymorphism modulates the relationship between dietary fat intake and components of the metabolic syndrome: results from the Quebec Family Study. Clin Genet 63:109-116

16. Memisoglu A, Hu FB, Hankinson SE et al (2003) Interaction between a peroxisome proliferator-activated receptor gamma gene polymorphism and dietary fat intake in relation to body mass. Hum Mol Genet 12:2923-2929

17. Luan J, Browne PO, Harding AH et al (2001) Evidence for gene-nutrient interaction at the PPARgamma locus. Diabetes 50:686-689

18. Marti A, Corbalan MS, Martinez-Gonzalez MA, Forga L, Martinez JA (2002) CHO intake alters obesity risk associated with Pro12Ala polymorphism of PPARgamma gene. J Physiol Biochem 58:219-220

19. Franks PW, Luan J, Browne PO et al (2004) Does peroxisome proliferator-activated receptor gamma genotype (Pro12ala) modify the association of physical activity and dietary fat with fasting insulin level? Metabolism 53:11-16

20. Valve R, Sivenius K, Miettinen R et al (1999) Two polymorphisms in the peroxisome proliferator-activated receptorgamma gene are associated with severe overweight among obese women. J Clin Endocrinol Metab 84:3708-3712
21. Meirhaeghe A, Fajas L, Helbecque N et al (1998) A genetic polymorphism of the peroxisome proliferator-activated receptor gamma gene influences plasma leptin levels in obese humans. Hum Mol Genet 7:435-440

22. Chao TH, Li YH, Chen JH et al (2004) The 161TT genotype in the exon 6 of peroxisome proliferator-activated receptor gamma gene is associated with premature acute myocardial infarction and increased lipid peroxidation in habitual heavy smokers. Clin Sci (Lond) 107:461-466

23. Wang XL, Oosterhof J, Duarte N (1999) Peroxisome proliferator-activated receptor gamma $\mathrm{C} 161 \rightarrow \mathrm{T}$ polymorphism and coronary artery disease. Cardiovasc Res 44:588-594

24. Meirhaeghe A, Fajas L, Gouilleux F et al (2003) A functional polymorphism in a STAT5B site of the human PPAR gamma 3 gene promoter affects height and lipid metabolism in a French population. Arterioscler Thromb Vasc Biol 23:289-294

25. Rieusset J, Seydoux J, Anghel SI et al (2004) Altered growth in male peroxisome proliferator-activated receptor gamma (PPARgamma) heterozygous mice: involvement of PPARgamma in a negative feed-back regulation of growth hormone action. Mol Endocrinol 18:2363-2377

26. Pihlajamaki J, Vanhala M, Vanhala P, Laakso M (2004) The Pro12Ala polymorphism of the PPAR gamma 2 gene regulates weight from birth to adulthood. Obes Res 12:187-190

27. Doney AS, Fischer B, Cecil JE et al (2003) Male preponderance in early diagnosed type 2 diabetes is associated with the ARE insertion/deletion polymorphism in the PPPIR3A locus. BMC Genet 4:11

28. Stephens M, Smith NJ, Donnelly P (2001) A new statistical method for haplotype reconstruction from population data. Am J Hum Genet 68:978-989

29. Zhao JH (2004) 2LD, GENECOUNTING and HAP: Computer programs for linkage disequilibrium analysis. Bioinformatics 20:1325-1326

30. Freeman JV, Cole TJ, Chinn S, Jones PR, White EM, Preece MA (1995) Cross sectional stature and weight reference curves for the UK, 1990. Arch Dis Child 73:17-24

31. Cole TJ, Freeman JV, Preece MA (1995) Body mass index reference curves for the UK, 1990. Arch Dis Child 73:25-29

32. Cole TJ, Bellizzi MC, Flegal KM, Dietz WH (2000) Establishing a standard definition for child overweight and obesity worldwide: international survey. BMJ 320:1240-1243

33. Rodriguez-Esparragon FJ, Rodriguez-Perez JC, Macias-Reyes A, Alamo-Santana F (2003) Peroxisome proliferator-activated receptor-gamma2-Pro12Ala and endothelial nitric oxide synthase- $4 \mathrm{a} / \mathrm{b}$ gene polymorphisms are associated with essential hypertension. J Hypertens 21:1649-1655

34. Ostgren CJ, Lindblad U, Melander O, Melander A, Groop L, Rastam L (2003) Peroxisome proliferator-activated receptorgammaPro12Ala polymorphism and the association with blood pressure in type 2 diabetes: Skaraborg Hypertension and Diabetes Project. J Hypertens 21:1657-1662

35. Ridker PM, Cook NR, Cheng S et al (2003) Alanine for proline substitution in the peroxisome proliferator-activated receptor gamma-2 (PPARG2) gene and the risk of incident myocardial infarction. Arterioscler Thromb Vasc Biol 23:859-863

36. Doney AS, Fischer B, Leese G, Morris AD, Palmer CN (2004) Cardiovascular risk in type 2 diabetes is associated with variation at the PPARG locus. A Go-DARTS study. Arterioscler Thromb Vasc Biol 24:2403-2407 